\title{
Alanine Aminotransferase to Aspartate Aminotransferase Ratio Measurement
}

National Cancer Institute

\section{Source}

National Cancer Institute. Alanine Aminotransferase to Aspartate Aminotransferase

Ratio Measurement. NCI Thesaurus. Code C106498.

The determination of the ratio of alanine aminotransferase (ALT) compared to aspartate aminotransferase (AST) present in a sample. The measurement may be expressed as a ratio or percentage. 